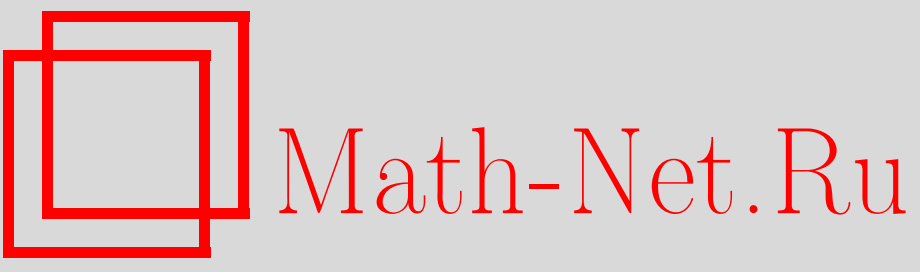

В. Н. Соловьев, K вопросу о минимаксно-байесовском оценивании, УМН, 1998, том 53, выпуск 5, 247-248

DOI: https://doi.org/10.4213/rm85

Использование Общероссийского математического портала Math-Net.Ru подразумевает, что вы прочитали и согласны с пользовательским соглашением

http://www.mathnet.ru/rus/agreement

Параметры загрузки:

IP : 54.162 .27 .143

26 апреля 2023 г., 16:54:33 


\title{
К ВОПРОСУ О МИНИМАКСНО-БАЙЕСОВСКОМ ОЦЕНИВАНИИ
}

\author{
В. Н. СолОвьЕВ
}

Обозначим через $\mathscr{P}$ класс всех распределений $P(y)$ случайного вектора $Y=\{\gamma, \theta\}$ с компонентами $\gamma \in \mathbb{R}^{n}, \theta \in \mathbb{R}^{m}$, имеющего известное среднее $\mathbb{E}_{P} Y=Y_{0}=\left\{\gamma_{0}, \theta_{0}\right\}$, но неизвестную ковариантную матрицу $K=\operatorname{cov}(Y, Y)$, которая принадлежит априорно заданному классу $\mathscr{K}$ симметрических матриц порядка $n+m$. Пусть по наблюдениям компоненты $\gamma$ нужно найти оценку $\hat{\ell}=\hat{\ell}(\gamma)$ для вектора $\ell=C \theta \in \mathbb{R}^{r}$. В качестве характеристики точности этой оценки примем величину

$$
D(\hat{\ell}):=\sup _{P \in \mathscr{P}} \mathbb{E}_{P}|\hat{\ell}-\ell|^{2}
$$

для отыскания оптимальной оценки $\hat{\ell}_{*}(\gamma)$ нужно на классе всех измеримых функций $\hat{\ell}: \mathbb{R}^{n} \rightarrow \mathbb{R}^{r}$ найти решение задачи

$$
D_{\min }:=\inf _{\hat{\ell}(\cdot)} D(\hat{\ell})
$$

Вместо величины $\mathbb{E}_{P}|\hat{\ell}-\ell|^{2}$ некоторые рассматривают $\mathbb{E}_{P}|\hat{\theta}-\theta|_{\Sigma}^{2}$, где $\|x\|_{\Sigma}^{2}=x^{T} \Sigma x$ и $\Sigma-$ заданная неотрицательно определенная матрица ранга $r \leqslant m$. Посколшку найдется разложение $\Sigma=C^{T} C$, в котором матрица $C \in \mathbb{R}^{r m}$ имеет ранг $r$, то $\|\hat{\theta}-\theta\|_{\Sigma}^{2}=|\hat{\ell}-\ell|^{2}$, где $\ell=C \theta$ и $\hat{\ell}=C \hat{\theta}$. Поэтому задача отыскания оптимальной оценки $\hat{\theta}_{*}$ сводится к задаче $(1)-(2)$.

В работе [1] были получены условия, при которых в задаче (1)-(2) существует седловая точка $\left\{\hat{\ell}_{*}, P_{*}\right\}$, и поэтому она эквивалентна двойственной задаче

$$
D_{\min }=\sup _{P \in \mathscr{P}} \inf _{\hat{\ell}(\cdot)} \mathbb{E}_{P}|\hat{\ell}-\ell|^{2}
$$

Распределение $P_{*} \in \mathscr{P}$, которое доставляет максимум в (3), назьвают наименее благоприятньг. Введем обозначение

$$
K=\left(\begin{array}{ll}
K_{\gamma \gamma} & K_{\gamma \theta} \\
K_{\theta \gamma} & K_{\theta \theta}
\end{array}\right), \quad \text { где } \begin{gathered}
K_{\gamma \gamma}=\operatorname{cov}(\gamma, \gamma) \\
K_{\gamma \theta}=\operatorname{cov}(\gamma, \theta)
\end{gathered}
$$

Sp $K$ - след матрицы $K ; K^{+}$- псевдообратная матрица (по Муру-Пенроузу).

Теорема 1. Если множсество $\mathscr{K}$ компактно, выпукло и состоит из неотрицательно определенных матрии, то

$$
D_{\min }=\max _{K \in \mathscr{K}} \operatorname{Sp} C\left(K_{\theta \theta}-K_{\theta \gamma} K_{\gamma \gamma}^{+} K_{\gamma \theta}\right) C^{T}
$$

Наименее благоприятным в априорном классе Я̆ является нормальное распределение $Y^{\sim} \mathbb{N}\left(Y_{0}, \bar{K}\right)$, в котором $\bar{K} \in \mathscr{K}$ - любое решение задачи (4). Решение исходной задачи (1)-(2) дает линейная оценка $\hat{\ell}_{*}$, которая в случае невырожденной матрицы $\bar{K}_{\gamma \gamma}$ имеет вид

$$
\hat{\ell}_{*}=C\left(\theta_{0}+\bar{K}_{\theta \gamma} \bar{K}_{\gamma \gamma}^{-1}\left(\gamma-\gamma_{0}\right)\right)
$$

Теорема 2. Если множество $\mathscr{K}$ компактно и состоит из неотрицательно определенных матрии, то все утверждения Теоремы 5 останутся справедливь, если выпукло не само әто множество, а матричное множество $\{\widetilde{K}: \exists K \in \mathscr{K}, K \geqslant \widetilde{K}\}$.

Теоремы 1 и 2 , которые являются обобщениями теоремы о нормалшной корреляции [2], получены в [3]. В случае, когда все распределения $P \in \mathscr{P}$ имеют известные среднее $Y_{0}$ и матрицу ковариаций $K$ или же множество $\mathscr{K}$ содержит максимальный элемент (т.е. существует матрица 
$\bar{K} \in \mathscr{K}$, такая что $K \leqslant \bar{K}$ при всех $K \in \mathscr{K})$, аналогичные резултаты для общих линейно-квадратичных задач получены (на язьке седловых точек) в работе V. D. Vanderlinde [4], уровень которой настолько высок, что ее автор не счел нужным обременять читателей общеизвестными формулами.

В указанных частных случаях линейная оценка $\hat{\ell}_{*}$ имеет вид [5]

$$
\hat{\ell}_{*}=C\left(\theta_{0}+\bar{K}_{\theta \gamma} \bar{K}_{\gamma \gamma}^{+}\left(\gamma-\gamma_{0}\right)\right)
$$

Но, к сожалению, в общем случае в теореме 1 нельзя просто заменить формулу (5) на (6). В качестве контрпримера можно взять

$$
\gamma_{1}=\theta_{1}+\theta_{2}+\rho_{1}, \quad \gamma_{2}=\theta_{1}+\rho_{2}, \quad \ell=\theta_{1}+\theta_{2} \quad(m=n=2, \quad r=1),
$$

где $\mathbb{E} \theta_{i}=\mathbb{E} \rho_{i}=0, \mathbb{E} \theta_{i}^{2} \leqslant 1, \mathbb{E} \rho_{i}^{2} \leqslant 1, i=1,2$, а взаимные ковариации 4 случайных величин $\theta_{1}$, $\theta_{2}, \rho_{1}, \rho_{2}$ совершенно произвольны. В этом случае будут выполнены условия теоремы 1 . Пусть $P_{*}$ - вырожденное нормальное распределение, в котором $\theta_{1}=-\varepsilon, \theta_{2} \equiv 0, \rho_{1}=\varepsilon, \rho_{2}=\varepsilon$, где $\varepsilon$-это стандартная гауссовская случайная величина. Покажем, что пара $\left\{\hat{\ell}_{*}, P_{*}\right\}$, в которой $\hat{\ell}_{*}=\gamma_{1}$, образует седловую точку в задаче (1)-(2). Действительно, поскольку $\hat{\ell}_{*}-\ell=\gamma_{1}-\left(\theta_{1}+\theta_{2}\right)=\rho_{1}$, то

$$
\sup _{P \in \mathscr{P}} \mathbb{E}_{P}\left(\hat{\ell}_{*}-\ell\right)^{2}=\sup _{P \in \mathscr{P}} \mathbb{E}_{P} \rho_{1}^{2}=\mathbb{E}_{P_{*}}\left(\hat{\ell}_{*}-\ell\right)^{2}=1
$$

Кроме того, для указанного распределения $P_{*}$ имеем $\gamma_{1} \equiv 0, \gamma_{2} \equiv 0, \ell=-\varepsilon$, и поэтому любая линейная оценка $\hat{\ell}(\gamma)$ постоянна: $\hat{\ell} \equiv \alpha$, откуда

$$
\inf _{\hat{\ell}(\cdot)} \mathbb{E}_{P_{*}}(\hat{\ell}-\ell)^{2}=\inf _{\hat{\ell}=\alpha} \mathbb{E}_{P_{*}}(\alpha+\varepsilon)^{2}=\mathbb{E}_{P_{*}}\left(\hat{\ell}_{*}-\ell\right)^{2}=1
$$

Равенства $(7),(8)$ по определению означают, что пара $\left\{\hat{\ell}_{*}, P_{*}\right\}$ - это седловая точка.

Тогда, как известно [3], $\hat{\ell}_{*}$ - это решение задачи (2), а $P_{*}-$ решение задачи (3). В то же время, поскольку для распределения $P_{*}$ имеем $\gamma_{1} \equiv 0, \gamma_{2} \equiv 0$, то в соответствуюшем ему решении $\bar{K}$ задачи (4) получим $\bar{K}_{\theta \gamma}=0, \bar{K}_{\gamma \gamma}=0$. Поэтому формула (6) дает оценку $\hat{\ell} \equiv 0$, которая не оптимальна, ибо согласно $(7)$ имеем $D\left(\hat{\ell}_{*}\right)=1$, тогда как

$$
D(\hat{\ell})=\sup _{P \in \mathscr{P}} \mathbb{E}_{P} \ell^{2}=\sup _{P \in \mathscr{P}} \mathbb{E}_{P}\left(\theta_{1}+\theta_{2}\right)^{2}=4
$$

Данньй пример возник в связи с теоремой 3.1 из [8], в которой (в чуть иных обозначениях) утверждалось, что для критерия $\sup _{P \in \mathscr{P}} \mathbb{E}_{P}\|\hat{\theta}-\theta\|_{\Sigma}^{2}$ и выпуклого компактного подмножества неотрицательно определенных матриц $\mathscr{K}$ максимальная оценка $\hat{\theta}_{*}$ имеет вид

$$
\hat{\theta}_{*}=\theta_{0}+\bar{K}_{\theta \gamma} \bar{K}_{\gamma \gamma}^{+}\left(\gamma-\gamma_{0}\right), \quad \bar{K} \in \arg \max _{K \in \mathscr{K}} \operatorname{Sp} \Sigma\left(K_{\theta \theta}-K_{\theta \gamma} K_{\gamma \gamma}^{+} K_{\gamma \theta}\right)
$$

\section{СПИСОК ЛИТЕРАТУРЫ}

[1] Соловьев В.Н. // УМН. 1995. Т. 50. №4. С. 171-172. [2] Липцер Р. Ш., Ширяев А. Н. Статистика случайных процессов. М.: Наука, 1974. [3] Соловьев В. Н. Двойственные экстремальные задачи и их применение к задачам минимаксного оценивания / / Автореферат дисс. . . д докт. физ.-матем. наук. М.: МИРАН, 1996. [4] Vanderlinde V. D. // IEEE Trans. on Automatic Control. 1977. V. AC 22. № 1. Р. 138-139. [5] Панков А. Р. // Технич. киберн. 1992. № 3. С. 63-70. [6] Соловьев В. Н. // УМН. 1996. Т. 51. № 3. С. 211-212. [7] Соловьев В. Н. // УМН. 1997. Т. 52. № 4. С. 49-86. [8] Панков А. Р. Минимаксные методы оценивания и оптимизации процессов в неопределенно-стохастических системах // Автореферат дисс. ... докт. физ.-матем. наук. М.: МАИ, 1998. 